\title{
An Integrated Game Theoretical Approach for Primary and Secondary Users Spectrum Sharing in Cognitive Radio Networks
}

\author{
Jong Gyu Kim ${ }^{\dagger}$, Khanh-Huy Nguyen ${ }^{++}$, Jungtae Lee ${ }^{+++}$, Won-Joo Hwang ${ }^{++++}$
}

\section{ABSTRACT}

\begin{abstract}
In this paper, we address the problem of bandwidth sharing among multiple primary users and multiple secondary users in a cognitive radio network. In cognitive radio networks, effective spectrum assignment for primary and secondary users is a challenge due to the available broad range of radio frequency spectrum as well as the requisition of harmonious coexistence of both users. To handle this problem, firstly, Bertrand game model is used to analyze a spectrum pricing in which multiple primary users emulate with each other to acquire maximal profit. After that, we employ Cournot game to model the spectrum sharing of secondary users to obtain optimal profit for each user also. Simulation results show that our scheme obtains optimal solution at Nash equilibrium.
\end{abstract}

Key words: Cognitive radio, Primary user, Secondary user, Spectrum sharing, Bertrand game, Cournot game.

\section{INTRODUCTION}

Recently, because of the increase in bandwidth demand of new wireless applications, radio frequency spectrum has become congested. Cognitive radio technology is one of promising candidates to supply high bandwidth for mobile users via diverse wireless architectures, and permits the secondary users to dynamically access the licensed bands

※ Corresponding Author: Won-Joo Hwang, Address: (621-749) Obangdong, Gimhae, Gyeongnam, Korea, TEL: +82-55-320-3847, FAX : +82-55-322-6275, E-mail : ichwang.inje.ac.kr

Receipt date: May 17, 2011, Revision date: Oot. 31, 2011 Approval date: Dec. 7, 2011

${ }^{+}$School of Computer Information, Yeungjin College, South Korea.

(E-mail : jkkim@yjc.ac.kr)

${ }^{+\dagger}$ Dept. of Information and Communications Engineering, HSV-TRC, Inje University, South Korea. (E-mail : huynkh@gmail.com)

${ }^{+++}$School of Computer Science and Engineering, Pusan National University. (E-mail : jtlee@pusan.ac.kr)

${ }^{++++}$Dept. of Information and Communications Engineering, HSV-TRC, Inje University, South Korea. used by primary users. Software defined radio technique and cognitive radio were presented in [1] to improve radio frequency spectrum utilization. An extensive survey about the functionalities and research challenges of cognitive radio networks was introduced in [2]. With cognitive radio technique, radio frequency spectrum can be shared between primary and secondary users. When the assigned spectrum of primary user (PU) is not entirely used, the primary user is willing to sell spectrum occasions to secondary services to enhance the usage of spectrum and also achieve higher revenue. The secondary users (SUs) then will share the spectrum that secondary services bought from primary users. However, because of the available broad range of radio frequency spectrum as well as the requisition of harmonious coexistence of both primary and secondary user, efficient dynamic spectrum allocation is really a challenge.

Game theory has been identified as one of key techniques for effective spectrum management of cognitive radio to attain the desired objectives, 
maximizing throughput and channel utilization [3] In [4], an introduction to game theory is described. In [5], a competitive pricing model for spectrum sharing using Bertrand game was proposed. A game theoretical adaptive channel allocation was presented in [6]. In [7], it introduced a multi-winner spectrum auction framework, and developed suitable mechanisms for that kind of auction. Cournot game is also used to model the problem of bandwidth allocation among SUs in cognitive radio networks [8]. In [9], a non-cooperative game-theoretic framework was proposed for radio resource management in $4 \mathrm{G}$ heterogeneous wireless access networks. A resource management method in wireless mesh network is provided in [10]. Using game theory, from existing works, they separated the bandwidth sharing into two cases: the spectrum allocation schemes for PUs and the spectrum allocation scheme for SUs. Regarding the spectrum allocation schemes for PUs, they focus on $\max ^{-}$ imizing profit of PUs and also determined the total bandwidth can be shared to SUs, but it does not concern about spectrum sharing among SUs to get optimal profit for SUs. On the other hand, the spectrum allocation schemes for SUs assume the total shared bandwidth from PUs without concerning whether that value is optimal for PUs or not. They focus on only spectrum assignment for SUs. Therefore, there is no significant relationship between the profit of PUs and profit of SUs which is meaningful and always required in real models. In this paper, we address the problem of bandwidth sharing among multiple primary users and multiple secondary users in a cognitive radio network. The purpose is to optimize both the profit of PUs and SUs within a rational connection between the spectrum sharing models, thus it also enhances spectrum utilization. In order to handle this problem, we propose a mixed game theoretical approach. Firstly, Bertrand game model is used to analyze a spectrum pricing in which a few primary users offer spectrum access occasions to a secon- dary service. We formulate this problem as an oligopoly market in which a few firms (i.e., PUs) emulate with each other in terms of price of commodity (i.e, spectrum) provided to the market to achieve maximal profit. In this case, for a PU, the bandwidth demand of the secondary service is presented based on a spectrum demand function and the cost of spectrum sharing is defined based on quality of service (QoS) attenuation. We consider the Nash equilibrium as the solution of this game. Obtaining that solution, we determine the aggregate spectrum sold for the secondary service. It is also the amount of available bandwidth that can be shared to SUs. Next, we employ Cournot game to model the spectrum sharing of secondary users. Here, we also formulate this problem as an oligopoly market where a few firms (i.e., SUs) compete with each other in terms of quantity of commodity to obtain optimal profit value. For each $\mathrm{SU}$, a revenue function is created by considering utility and the cost is modeled based on the loss of QoS and payment for using spectrum within a relationship with the result of the previous Bertrand game model. The Nash equilibrium is regarded as a solution of this game. We utilize the iterative algorithm to obtain the Nash equilibrium.

The rest of this paper is organized as follows. Section 2 describes the system model and assumptions. In Section 3, we present game models and formulate the problem. Simulation results are shown in Section 4, and Section 5 concludes the paper.

\section{SYSTEM MODEL}

We consider a cognitive radio network with $M$ primary users and a secondary service serving $N$ secondary users as shown in Figure 1. In this case, primary user $i$ operating on the frequency spectrum $F_{i}$ tends to sell its unused bandwidth (e.g. time slots in TDMA or radio frequencies in FDMA) at price $p_{i}$ (per unit of bandwidth) to the secondary 


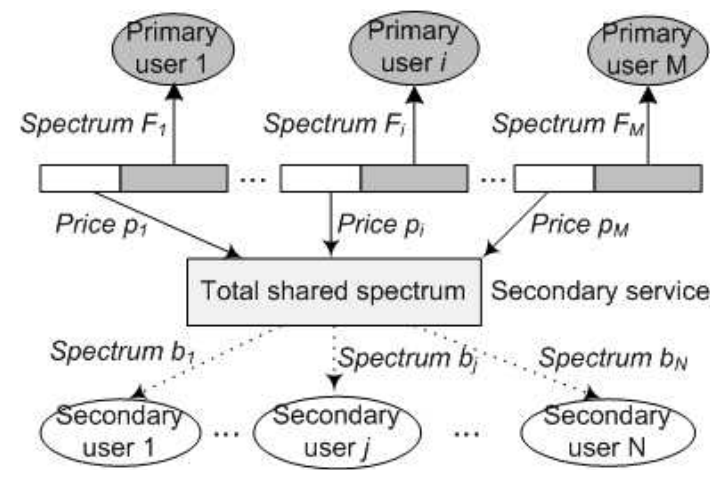

Fig. 1. System model for spectrum sharing.

service for monetary gains. The spectrum then can be shared for $N$ secondary users. Each secondary user $i$ is allocated a portion of the total shared spectrum $b_{i}$. After spectrum assignment, the secondary users use adaptive modulation to convey in the assigned spectrum in order to improve efficiency of the transmission.

Using adaptive modulation, transmission rate of users is dynamically adjusted based on the transmission channel quality. We can compute the spectral efficiency $k$ of the transmission for users as follows [5, (1)]. In order to guarantee QoS for users, the bit error rate (BER) must be maintained less than or equal to the target BER level $\left(B E R^{\text {tar }}\right)$. By channel evaluation, we suppose that the SNR information is available at the receiver.

\section{SPECTRUM SHARING MANAGEMENT SCHEME}

In this section, we formulate the spectrum sharing problem among PUs and SUs as oligopoly markets. We first utilize Bertrand game to model a spectrum pricing of PUs. And then, a Cournot game model is proposed to handle the problem of spectrum assignment for SUs.

\subsection{Cigopdy Market Competition, Bertrand and Cournot game}

In cognitive radio networks, radio frequency spectrum can be shared among primary users (licensed users) and secondary users (unlicensed users) to increase spectrum usage and also to gain profit for primary users (spectrum owners). Hence, an economic model would be suitable for primary users and secondary users to effectively share the spectrum. We will describe the spectrum sharing problem as oligopoly market competitions in which a number of firms compete with each other independently to obtain the maximum profit by governing quantity or price of commodity provided to the market. The oligopoly market is a kind of market where a small number of firms control a particular market. In this market structure, action of one firm may be observed by other firms and the decision of each firm is affected by actions of other firms. This situation can be modelled using Bertrand and Cournot game models.

Let's repeat basic concept about Bertrand and Cournot game models. These game models consider a competition between two firms produce a same product. The cost of each firm to make product is constant and each firm wants to maximize its profit by control price or quantity of product. The profit function is total revenue (the price multiply by the quantity) minus total costs. In Bertrand model, the profit is formulated as a function of price: $U_{i}=f\left(P_{i}\right)$ with $P_{i}$ is price of product, $i=1,2$. Then each firms will manage its price to maximize its profit. By contrast, in Cournot model, the profit is formulated as a function of quantity: $U_{i}=f\left(Q_{i}\right)$, with $Q_{i}$ is quantity of product. Each firms will control its quantity to get the optimal profit.

When primary users do not use fully the allocated spectrum, they are willing to share its spectrum to secondary users to get more revenue (thus profit). This can be considered as a trading competition where there are many primary users (sellers) offer spectrum to the secondary service (buyer). The question is how to determine the spectrum price of each primary user to maximize the profit. To handle this question, therefore, a 
Bertrand game model is well suit with price emulation to attain the optimal pricing strategy for PUs. In this game, the players are PUs. The players compete with each other in terms of the price of commodity. The strategy of each player $i$ is the price per unit of bandwidth (represented by $p_{i}$ ) which is non-negative. The payoff of each player $i$ is the profit (i.e., revenue minus cost) denoted by $U_{i}^{P}$. On the other hand, after determining the shared spectrum from PUs to the secondary service, SUs will share together this spectrum quantity. The problem here is how to set the spectrum size for each SU to optimize the profit of each SU. Accordingly, a Cournot game model is compatible with spectrum sharing problem among SUs. In this game, the players are SUs. The players compete with each other in terms of requested spectrum size, i.e., the quantity of commodity. The strategy of each secondary user $j$ corresponds to the allocated spectrum size (represented by $b_{j}$ ) which is also non-negative. The profit of each player $j$ (represented by $U_{j}^{S}$ ) can be calculated from the cost and the revenue gained from utilization the allocated spectrum as well.

\subsection{Spectrum Pricing Competition Scheme for Primary Users}

For each primary user $i$, the spectrum is sold to the secondary service is the spectrum demand for frequency spectrum $F_{i}$ at the secondary service. The spectrum demand function $D_{i}(p)$ of secondary service can be described from [5] as follows:

$$
\begin{aligned}
D_{i}(p) & =D_{1}\left(p_{-i}\right)-D_{2} p_{i} \\
\text { with } D_{1}\left(p_{-i}\right) & =\frac{k_{i}^{(s)}(\nu(M-2)+1)-\nu \sum_{i \neq j}\left(k_{j}^{(s)}-p_{j}\right)}{(1-\nu)(\nu(M-1)+1)} \\
D_{2} & =\frac{\nu(M-2)+1}{(1-\nu)(\nu(M-1)+1)}
\end{aligned}
$$

where $k_{i}^{(s)}$ denotes the spectral efficiency of transmission of a SU utilizing spectrum $F_{i}, \nu \in[-1.0,1.0]$ denotes the spectrum substitutability parameter and $p=\left\{\mathrm{p}_{1}, \ldots, \mathrm{p}_{\mathrm{M}}\right\} \quad$ denotes the set of prices offered by PUs, $p=p_{-i} \cup\left\{\mathrm{p}_{\mathrm{i}}\right\}$.

The revenue of each PU includes benefits from selling a portion of its spectrum and utilization its remaining spectrum. We can formulate the benefit of utilization the remaining spectrum based on considering it is as capacity of the bandwidth Thus, Shannon formula is applied to do this. Therefore, the revenue function $R_{i}^{(p)}$ for primary user $i$ is described as follows:

$$
R_{i}^{(p)}=p_{i} D_{i}(p)+c_{1}\left(W_{i}-D_{i}(p)\right) \log \left(1+\gamma_{i}^{(p)}\right)
$$

where $c_{1}$ denotes the constant weights, $W_{i}$ is the size of allocated spectrum for PU $i, \gamma_{i}^{(p)}$ is the SNR at the receiver of PU $i$.

In order to define the cost function, we consider the QoS degradation of the PUs. Sharing a portion of the spectrum with the secondary service may make attenuation in the QoS performance of the PUs. If the required bandwidth for primary connections to the PU is insufficient, the PU has to "compensate" to the connections, and this is regarded as the cost of spectrum sharing with secondary service. The cost function $C_{i}^{(p)}$ for primary user $i$ is expressed as follows:

$$
C_{i}^{(p)}=c_{2}\left(B_{i}^{r e q(p)}-k_{i}^{(p)}\left(W_{i}-D_{i}(p)\right)\right)^{2}
$$

where $c_{2}$ denotes the constant weight for the cost function, $B_{i}^{r e q(p)}$ is the required bandwidth for primary connections, $k_{i}^{(p)}$ is the spectral efficiency of PU $i$. Consequently, the pofit function for PU $i$ can be obtained as follows:

$$
\begin{aligned}
U_{i}^{P}(p)= & p_{i} D_{i}(p)+c_{1}\left(W_{i}-D_{i}(p)\right) \log \left(1+\gamma_{i}^{(p)}\right) \\
& -c_{2}\left(B_{i}^{r e q(p)}-k_{i}^{(p)}\left(W_{i}-D_{i}(p)\right)\right)^{2} .
\end{aligned}
$$

Thus, we find the optimal solution to maximize the profit of each primary user as follows:

$$
\begin{array}{ll}
\text { Max } & U_{i}^{P}(p) \\
\text { S.t. } & p_{i}>0 \forall i .
\end{array}
$$

The Nash equilibrium was defined in [4] as a strategy profile in which no player can increase his profit, given actions of other players. In this game, 
the Nash equilibrium is achieved based on the best response function that is the best strategy of one player given strategies of other players. The best response function of $\mathrm{PU} i$ is defined as follows:

$$
B R_{i}\left(p_{-i}\right)=\arg \max _{p_{i}>0} U_{i}^{P}(p) .
$$

The set $p^{*}=\mathrm{p}_{1}^{*}, \mathrm{p}_{2}^{*}, \ldots, \mathrm{p}_{\mathrm{M}}^{*}$ denotes the Nash equilibrium of this game if and only if

$$
p_{k}^{*}=B R_{k}\left(P_{-k}^{*}\right) \quad \forall k .
$$

Mathematically, we can acquire the Nash equilibrium by solving the following set of equations:

$$
\begin{aligned}
& \frac{\partial U_{i}^{P}(p)}{\partial p_{i}}=0 \text { for all } i \\
& \quad D_{1}\left(p_{-i}\right)-2 D_{2} p_{i}+c_{1} D_{2} \log \left(1+\gamma_{i}^{(p)}\right) \\
& +2 c_{2} k_{i} D_{2}\left(B_{i}^{r e q(p)}-k_{i}^{(p)}\left(W_{i}-\left(D_{1}\left(p_{-i}\right)-D_{2} p_{i}\right)\right)\right)=0 .
\end{aligned}
$$

When all parameters in (1) are given, the optimal solution $p^{*}$ can be achieved by solving the above set of linear equations. Based on $p^{*}$, we obtain the size of the shared spectrum from the spectrum $\mathrm{de}^{-}$ mand functions $D_{i}\left(p^{*}\right)$. For the case of $\mathrm{M}=2$, the set of equations in (1) can be described as in (2) and (3).

\subsection{Spectrum Sharing Scheme for Secondary Users}

In order to quantify the revenue of secondary users, we employ the concept of utility to denote the revenue gained from an allocated bandwidth. The utility function $R_{j}^{(s)}$ (i.e., the revenue) of secondary user $j$ assigned a frequency spectrum $b_{j}$ from the secondary service is defined as follows [11]:

$$
R_{j}^{(s)}=c_{3} \log \left(1+\omega b_{j}\right)
$$

where $c_{3}$ and $\omega$ are constants indicating the scale and the shape of the utility function, respectively.

The cost function of a secondary user is considered based on the payment of the secondary user for using the shared spectrum and the the attenu- ation in the QoS performance. Because SUs do not always must utilize fully the shared spectrum, we define the cost per unit of bandwidth which the secondary has to pay is $\bar{p}+\alpha_{j} \sum_{j=1}^{N} b_{j}$ where $\bar{p}=\sum_{i=1}^{M} D_{i}\left(p^{*}\right) p_{i}^{*} / \sum_{i=1}^{M} D_{i}\left(p^{*}\right) \quad$ is average cost per unit of bandwidth which the secondary bought from PUs given from solving (1) and $\alpha_{j}$ is non-negative constant weight corresponding to each SU. Besides, if the frequency spectrum allocated to the SU is not enough for secondary connections to the SU, this is also considered as the lost of QoS performance. Hence, this degradation is a part of the cost function. Therefore, the cost function $C_{j}^{(s)}$ for $\mathrm{sec}^{-}$ ondary user $j$ is shown as follows:

$$
C_{j}^{(s)}=c_{4}\left(k_{j}^{(s)} b_{j}-B_{j}^{r e q(s)}\right)^{2}+\left(\bar{p}+\alpha_{j} \sum_{j=1}^{N} b_{j}\right) b_{j}
$$

where $c_{4}$ is constant weight, $B_{j}^{r e q(s)}$ is the required bandwidth for secondary connections, $k_{j}^{(s)}$ is the spectral efficiency of SU $j$. Consequently, the pofit function for SU $j$ can be obtained as follows:

$$
\begin{aligned}
U_{j}^{S(b)=} & c_{3} \log \left(1+\omega b_{j}\right)-c_{4}\left(k_{j}^{(s)} b_{j}-B_{j}^{r e q(s)}\right)^{2} \\
& -\left(\bar{p}+\alpha_{j} \sum_{j=1}^{N} b_{j}\right) b_{j}
\end{aligned}
$$

where $b=\left\{\mathrm{b}_{1}, \ldots, \mathrm{b}_{\mathrm{N}}\right\}$ denotes the set of spectrum allocated to SUs. We represent $D=\sum_{i=1}^{M} D_{i}\left(p^{*}\right)$ is the size of the total shared spectrum, our objective function for the spectrum sharing problem of the secondary user is

$$
\begin{array}{ll}
\text { Max } & U_{j}^{S(}(b) \\
\text { S.t. } & b_{j} \leq D-\sum_{j \neq i} b_{i} \\
& b_{j} \geq 0 \quad \forall j .
\end{array}
$$

Here, we also consider the Nash equilibrium as a solution of this game and use the best response function to obtain the Nash equilibrium. We define the best response function of $\mathrm{SU} j$ as follows:

$$
B R_{j}\left(b_{-j}\right)=\arg \max _{b_{j} \in X_{j}} U_{j}^{S}(b)
$$

where $b=b_{-j} \cup\left\{b_{j}\right\}$ and $X_{j}=\left[0, D-\sum_{j \neq i} b_{i}\right]$. Similarly, the set $b^{*}=\left\{\mathrm{b}_{1}^{*}, \mathrm{~b}_{2}^{*}, \ldots, \mathrm{b}_{\mathrm{N}}^{*}\right\}$ denotes the Nash equili- 


$$
\begin{aligned}
& \frac{1}{1-v^{2}}\left(2+\frac{2 c_{2}\left(k_{1}^{(p)}\right)^{2}}{1-v^{2}}\right) p_{1}-\frac{v}{1-v^{2}}\left(1+\frac{2 c_{2}\left(k_{1}^{(p)}\right)^{2}}{1-v^{2}}\right) p_{2} \\
& =\frac{k_{1}^{(s)}-v k_{2}^{(s)}}{1-v^{2}}+\frac{c_{1} \log \left(1+\gamma_{1}^{(p)}\right)}{1-v^{2}}+\frac{2 c_{2} k_{1}^{(p)}}{1-v^{2}}\left(B_{1}^{r e p(p)}-k_{1}^{(p)} W_{1}+\frac{k_{1}^{(p)} k_{1}^{(s)}}{1-v^{2}}-\frac{v k_{1}^{(p)} k_{2}^{(s)}}{1-v^{2}}\right) \\
& \frac{1}{1-v^{2}}\left(2+\frac{2 c_{2}\left(k_{2}^{(p)}\right)^{2}}{1-v^{2}}\right) p_{2}-\frac{v}{1-v^{2}}\left(1+\frac{2 c_{2}\left(k_{2}^{(p)}\right)^{2}}{1-v^{2}}\right) p_{1} \\
& =\frac{k_{2}^{(s)}-v k_{1}^{(s)}}{1-v^{2}}+\frac{c_{1} \log \left(1+\gamma_{2}^{(p)}\right)}{1-v^{2}}+\frac{2 c_{2} k_{2}^{(p)}}{1-v^{2}}\left(B_{2}^{r e p(p)}-k_{2}^{(p)} W_{1}+\frac{k_{2}^{(p)} k_{2}^{(s)}}{1-v^{2}}-\frac{v k_{2}^{(p)} k_{1}^{(s)}}{1-v^{2}}\right)
\end{aligned}
$$

brium of this game if and only if

$$
b_{k}^{*}=B R_{k}\left(b_{-k}^{*}\right) \quad \forall k \text {. }
$$

Mathematically, we can obtain the Nash equilibrium by solving the following set of equations: $\frac{\partial U_{j}^{S}(b)}{\partial b_{j}}=0$ for all $j$. However, we can see that $\mathrm{re}^{-}$ solving the above set of equations to achieve the Nash equilibrium is quite complex because these equations are not linear. In this paper, we utilize the iterative algorithm [9]. We begin with the starting points $b_{2}^{s t}, b_{3}^{s t}, \ldots, b_{n}^{s t}$. We compute new variables in the next iteration based on the current variables and the algorithm is finished when the difference between the next variables and the current variables is less than the limitary value $\varepsilon$.

\section{NUMERICAL ANALYSIS}

\subsection{Spectrum Pricing Competition of Primary Users}

We consider the case of a cognitive radio environment with two primary users and a secondary service. We create parameter set based on the parameter setting in [5]. The target BER for users is $B E R^{t a r}=10^{-4}$. The required bandwidth for primary connections of each PU is $B_{i}^{r e q(p)}=2 \mathrm{Mbps}$. We assume the frequency spectrum allocated to each PU is $W_{i}=2 \mathrm{MHz}$ and the weight constants $c_{1}=10, c_{2}=1$. The SNR at the receiver of users change from 9 to $22 \mathrm{~dB}$ and the spectrum substitutability parameter varies from 0.1 to 0.6 . We assume $\gamma_{1}^{(p)}=20 \mathrm{~dB}, \gamma_{2}^{(p)}=19 \mathrm{~dB}, \gamma_{1}^{(s)}=\gamma_{2}^{(s)}=10$ $\mathrm{dB}$ and $\nu=0.4$ in the main case.

We first evaluate variance of the profit of the PU with offered price. Figure 2 shows that the profit of the PU is a function of offered price. When the offered price increases, the profit first increases because there is more the generated revenue corresponding to higher price. Until a certain value, the profit decrease due to reduction of bandwidth $\mathrm{de}^{-}$ mand from the secondary service. The price make the maximal profit is the best response. Hence, we can find the optimal offered price of a PU given the prices of other PUs. Moreover, we also see that a larger offered price of PU2 results in a higher optimal price as well as a little higher the maximal profit value of PU1. That is because of when the offered price of PU2 increases, the bandwidth demand to PU1 is increased. Therefore, PU1 may offer the higher price to obtain the higher profit.

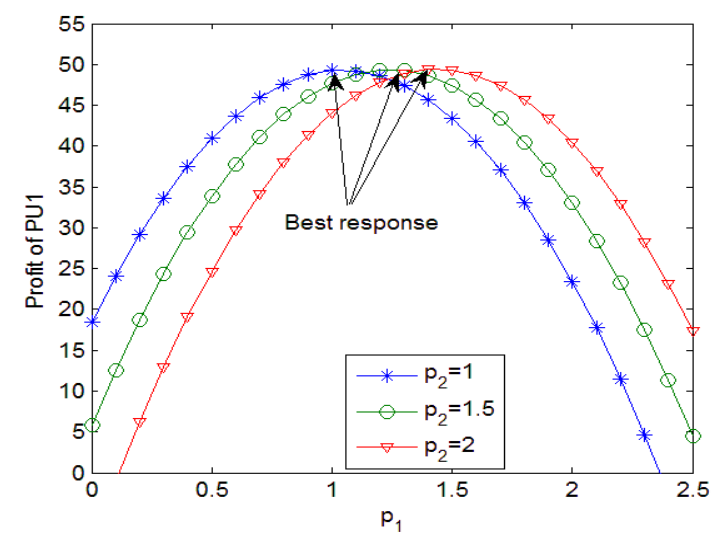

Fig. 2. Profit of $\mathrm{PU}_{1}$ vs. offered price. 


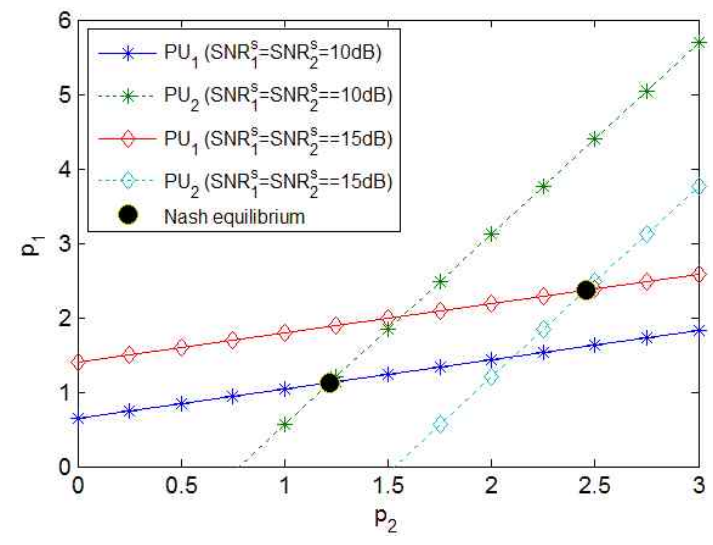

Fig. 3. Nash equilibrium and Best response functions.

The Nash equilibrium and the best response functions of PUs are shown in Figure 3 under different channel quality represented through different SNR values of the secondary service. The Nash equilibrium is indicated in which the best response functions of PUs intersect. When the channel quality becomes better (i.e., the SNR value of the secondary service is higher), the bandwidth demand is increased. As a result, PUs can offer the higher price.

Figure 4 and 5 show the price and the profit of both PUs at the Nash equilibrium under different channel qualities corresponding to PU1. When the channel quality corresponding to PU1 becomes better, the bandwidth demand to PU1 is higher as well. Hence, PU1 can offer the higher price and amount of sharing spectrum in order to obtain the higher profit. Because of the smaller bandwidth demand,

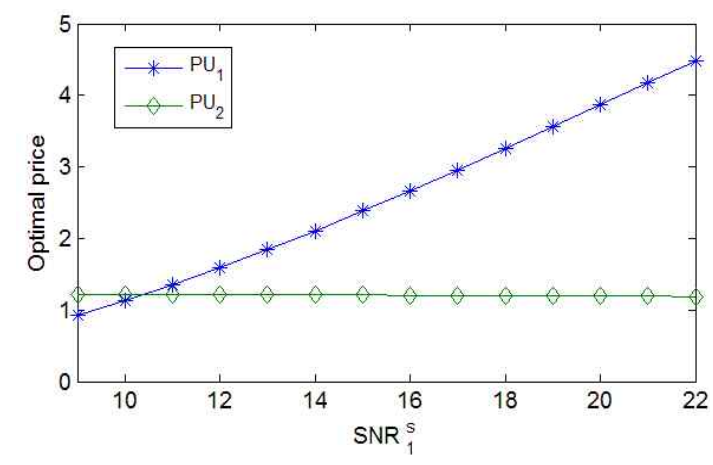

Fig. 4. Optimal price under different channel qualities corresponding to $\mathrm{PU}_{1}$.

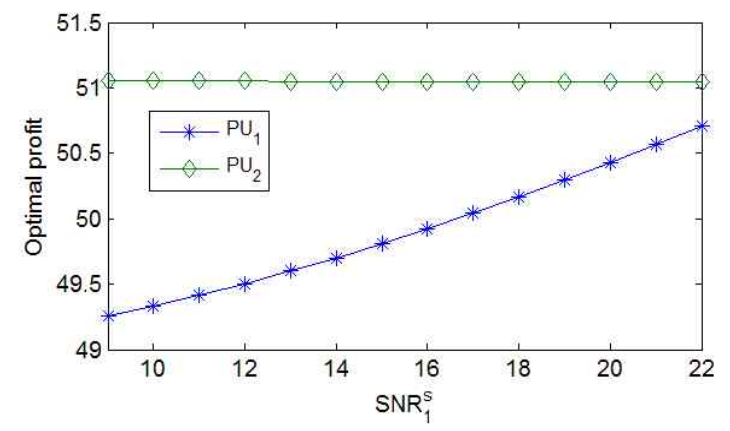

Fig. 5. Optimal profit under different channel qualities corresponding to $\mathrm{PU}_{1}$.

the price and the profit of PU2 are mitigated when the price and the profit of PU1 are gained.

\subsection{Spectrum Sharing Competition of Secon- dary Users}

We consider the case of a cognitive radio environment with two secondary users sharing a frequency spectrum given from spectrum pricing competition of primary users. This size of the total shared spectrum is $D=0.5663 \mathrm{MHz}$ and $\bar{p}=1.1638$ The target BER for both SUs is $B E R^{t a r}=10^{-4}$. The required bandwidth for secondary connections of each SU is $B_{1}^{r e q(s)}=0.4 \mathrm{Mbps}, B_{2}^{r e q(s)}=0.3 \mathrm{Mbps}$. The parameters of the profit function are used as follows: $c_{3}=c_{4}=1, \omega=1000, \alpha_{1}=2, \alpha_{2}=3$. The SNR at the receiver of SUs also change from 9 to $22 \mathrm{~dB}$. In this case, we assume $\gamma_{1}^{(s)}=12 \mathrm{~dB}, \gamma_{2}^{(s)}$ $=10 \mathrm{~dB}$. The limitary value is $\varepsilon=10^{-7}$.

Let's first consider the profit function of a secondary user under different strategies adopted by another secondary user in Figure 6. When bandwidth offered by SU1 increases, as expected, the profit of SU1 increases due to generating more utility. However, after a certain point, this profit is decreased. That is because the cost is higher and higher when the offered bandwidth increases. The maximum profit of SU1 can be determined, given the strategy of SU2, and the strategy corresponding to this optimal value is considered as the best response for SU1. While the bandwidth offered by 


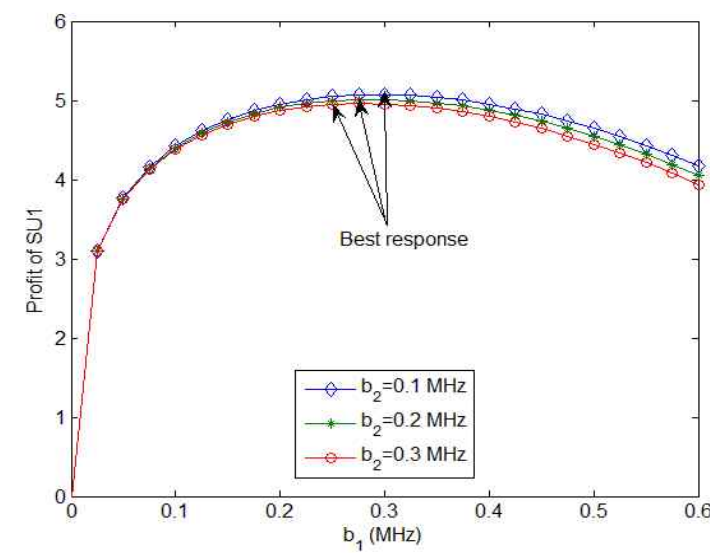

Fig. 6. The profit function and best response of $\mathrm{SU}_{1}$ under different offered spectrum of $\mathrm{SU}_{2}$.

SU2 increases, the bandwidth SU1 can offer is decreased. As a result, SU1 achieve the smaller best response value as well as the smaller profit. Figure 7 shows the best responses of both secondary users. The Nash equilibrium is located at the point that the best responses of the secondary users intersect. We also see that the Nash equilibrium is indicated at the different points under different channel qualities.

We next estimate the adaptation of the Nash equilibrium. Figure 8 and 9 show the offered spectrum and the profit of both SUs at the Nash equilibrium under different channel qualities. When the channel quality becomes better, the spectral efficiency is also gained. Therefore, in order to reduce

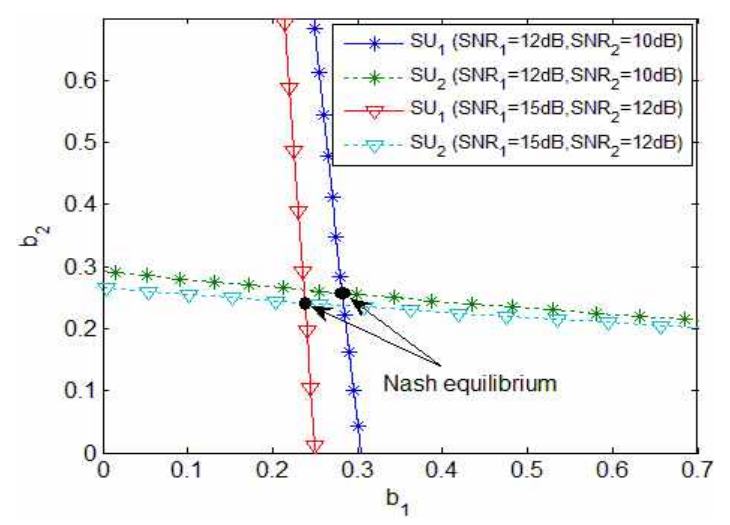

Fig. 7. Nash equilibrium and Best responses.

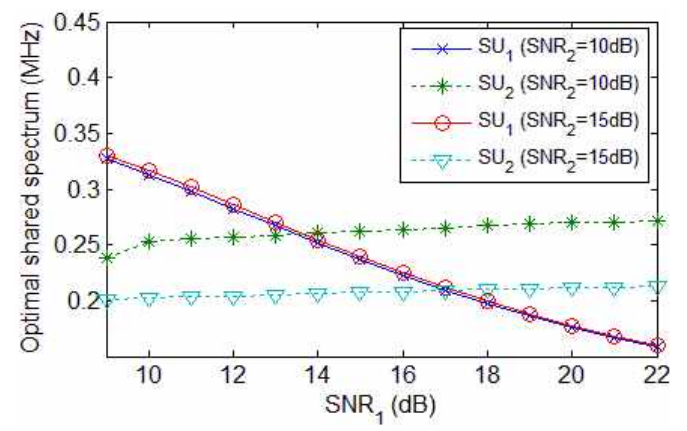

Fig. 8. Nash equilibrium of spectrum sharing under different channel qualities.

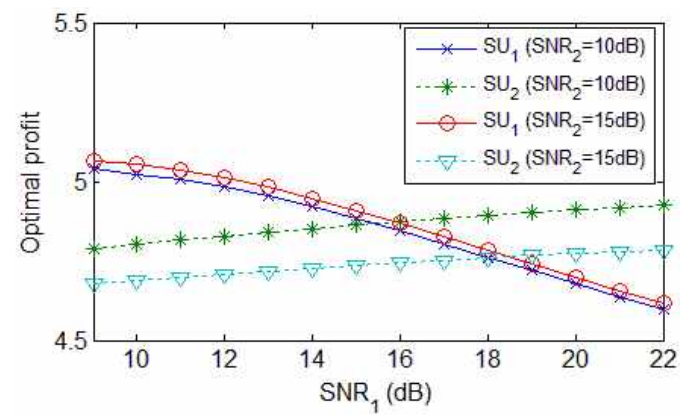

Fig. 9. Optimal profit under different channel

attenuation in QoS performance, the spectrum allocated to the secondary user is decreased. As a result, the profit is lessened as well. We also observe that the channel quality of a secondary user affects the assigned spectrum size for other secondary user.

To estimate the iterative algorithm, we reveal its convergence to the optima in some cases of different channel qualities in Figure 10. In this simulation, we choose the starting point is $100 \mathrm{Kbps}$. By using the iterative algorithm, the optimal value converges to a certain value just in several iterations as shown in Figure 10. We observe that the iterative algorithm may reach the optimal solution quite fast and smoothly.

\section{CONCLUSION}

In this paper, we consider the problem of bandwidth sharing among multiple primary users and multiple secondary users in a cognitive radio 


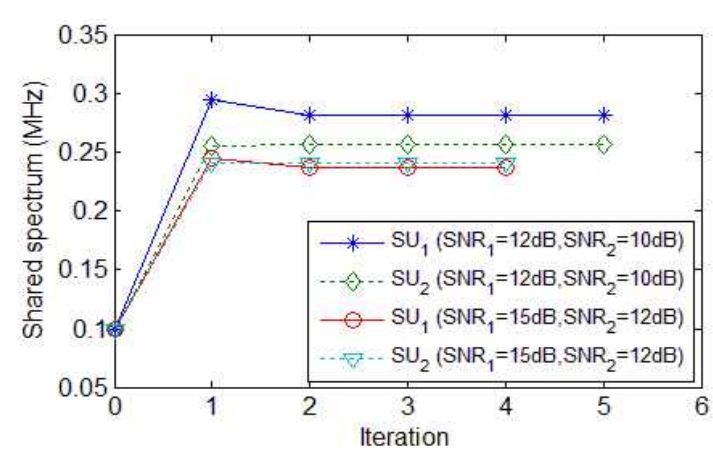

Fig. 10. Convergence of the iterative algorithm.

network. We propose an integrated game theoretical approach to handle the effective spectrum assignment. Firstly, Bertrand game model is utilized to analyze a spectrum pricing in which multiple primary users emulate with each other to acquire maximal profit through offering spectrum access opportunities to secondary users. We next use Cournot game to model the spectrum sharing of secondary users to obtain optimal profit value for each secondary user also. We formulate these problems as oligopoly market emulations. Nash equilibrium is regarded as a solution of each game. In the spectrum sharing among secondary users, the iterative algorithm is proposed to find the Nash equilibrium.

\section{ACKNOWLEDGMENTS}

This research was supported by Basic Science Research Program through the National Research Foundation of Korea(NRF) funded by the Ministry of Education, Science and Technology(2011-0024888).

\section{REFERENCES}

[1] J. Mitola, "Cognitive Radio for Flexible Multimedia Communications," MoMuC 1999, pp.3-10, 1999.

[2] I. F. Akyildiz, W. Y. Lee, M. C. Vuran, and S. Mohanty, "NeXt Generation/dynamic Spectrum Access/cognitive Radio Wireless Networks: A Survey," J. of Computer Networks,
Vol.50, pp.2127-2159, 2006.

[3] S. Haykin, "Cognitive Radio: Brain-Empowered Wireless Communications," IEEE J. on Selected Areas in Communications, Vol.23, No.2, pp.201-220, 2005.

[4] M. J. Osborne, An Introduction to Game Theory, Oxford University Press, 2003.

[5] D. Niyato and E. Hossain, "Competitive Pricing for Spectrum Sharing in Cognitive Radio Networks: Dynamic Game, Inefficiency of Nash Equilibrium, and Collusion," IEEE J. on Selected Areas in Communications, Vol.26, No.1, pp.192-202, 2008.

[6] N. Nie and C. Comaniciu, "Adaptive Channel Allocation Spectrum Etiquette for Cognitive Radio Networks," IEEE DYSPAN 2005, pp.269-278, 2005.

[7] Yongle Wu, Beibei Wang, K. Liu and T.C. Clancy, "A Multi-Winner Cognitive Spectrum Auction Framework with Collusion-Resistant Mechanisms," IEEE DySPAN 2008, pp.1-9, 2008.

[8] D. Niyato and E. Hossain, "A Game-Theoretic Approach to Competitive Spectrum Sharing in Cognitive Radio Networks," IEEE WCNC 2007. pp.16-20, 2007.

[9] D. Niyato and E. Hossain, "A Noncooperative Game-Theoretic Framework for Radio Resource Management in 4G Heterogeneous Wireless Access Networks," IEEE Trans. on Mobile Computing, Vol.7, No.3, pp.332-345, 2008.

[10] N. Pham, M. Choi and W. Hwang, "Joint Channel Assignment and Multi-path Routing in Multi-radio Multi-channel Wireless Mess Network," $J$. of The Korea Multimedia Society, Vol.12, no.6, pp.824-832, June 2009.

[11] H. Shen and T. Basar, "Differentiated Internet pricing Using a Hierarchical Network Game Model," IEEE ACC 2004, Vol.3, pp. 2322-2 327, 2004. 


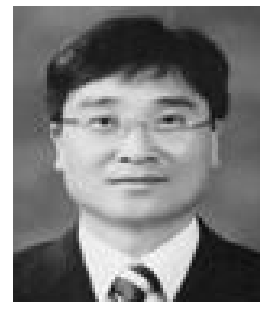

Jong-Gyu Kim

He is currently the Director of IT Center at Yeungjin College, Daegu City. From 2001 to now, he worked at the same school as Professor. He received his B.S. and M.S. degrees in Computer Engineering and is a Ph.D. candidate in Computer Engineering at Pusan National University.

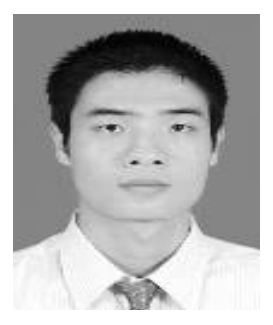

\section{Khanh-Huy Nguyen}

He received his bachelor's de ${ }^{-}$ gree in Electronic and Telecommunications from Hanoi University of Science and Technology, Vietnam in 2009. He is now Master student of the Department of Electronic and Telecommunications Engineering, Inje University, Gimhae, Republic of Korea. His research interests are optical network optimization and resource management in cognitive radio network.

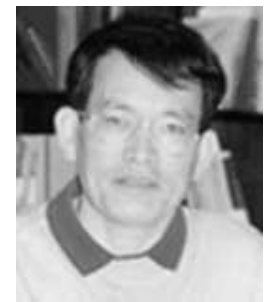

Jung-Tae Lee

Currently, he is a Professor at the Department of Computer Engineering in Pusan National University. He received his B.S. degree in Electronics from Pusan National University. He received his M.S. and Ph.D. in Computer Engineering from Seoul National University.

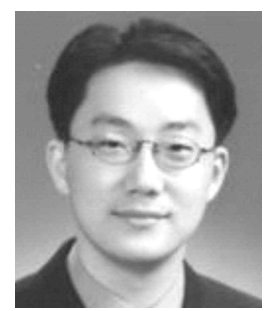

\section{Won-Joo Hwang}

He received the Ph.D Degree from Osaka University Japan in 2002. He received his bachelor's degree and M.S. degree in $\mathrm{Com}^{-}$ puter Engineering from Pusan National University, Pusan, Republic of Korea, in 1998 and 2000. Since September 2002, he has been an assistance professor at Inje University, Republic of Korea. His research interests are in Network Optimization and Ubiquitous Sensor Networks. 